\title{
AR SYSTEMS AND AR PROCESSES: THE SINGULAR CASE*
}

\author{
M. DEISTLER ${ }^{\dagger}$, A. FILLER ${ }^{\ddagger}$, AND B. FUNOVITS $\$$
}

Abstract. We deal with singular multivariate AR systems and the corresponding AR processes. An AR system is called singular if the variance of the white noise innovation is singular. AR processes are the stationary solutions of AR systems. In the singular case AR processes consist of a linearly regular and a linearly singular component. The corresponding Yule-Walker equations and in particular the possible non-uniqueness of their solutions are discussed. A particular canonical form is presented. Singular AR systems naturally arise as models for latent variables in dynamic factor analysis.

1. Introduction. Autoregressive (AR) systems and autoregressive systems with exogenous variables (ARX) are perhaps the most widely used model classes in time series analysis. Here we deal with the multivariate AR case. Whereas it is usually assumed that the one-step-ahead prediction error variance is non-singular, the focus of this paper is on the case where this variance is singular. This singular case has been discussed in detail in [11], [4], [8], [3].

We consider AR systems

$$
a(z) y_{t}=\nu_{t}
$$

where $y_{t}$ are observed $s$-dimensional outputs, $z$ is a complex variable as well as the backward-shift on the integers $\mathbb{Z}$, i.e. $z\left(y_{t} \mid t \in \mathbb{Z}\right)=\left(y_{t-1} \mid t \in \mathbb{Z}\right)$,

$$
a(z)=I-a_{1} z-\ldots-a_{p} z^{p}, \quad a_{j} \in \mathbb{R}^{s \times s}
$$

and $\left(\nu_{t} \mid t \in \mathbb{Z}\right)$ is an unobserved $s$-dimensional stochastic process. Throughout we assume that

(A1) $a_{0}$ is non-singular

(A2) $\nu_{t}$ is white noise, i.e.

- $\mathbb{E} \nu_{t}=0$

- $\Sigma=\mathbb{E} \nu_{t} \nu_{t}^{\prime}$ does not depend on $t$

- $\mathbb{E} \nu_{s} \nu_{t}^{\prime}=0, s \neq t$ and that

(A3) $\nu_{t}$ are the one-step-ahead forecast errors, i.e. $\mathbb{E} y_{s} \nu_{t}^{\prime}=0$ for $s<t$.

\footnotetext{
* Dedicated to Brian Anderson on the occasion of his 70th birthday. Support by the FWF (Austrian Science Fund under contract P20833/N18) and the ARC (Australian Research Council under Discovery Project Grant DP1092571) is gratefully acknowledged.

${ }^{\dagger}$ Research Group of Econometrics and System Theory, Department of Mathematical Methods in Economics, Vienna UT, and Institute for Advanced Studies, Vienna, E-mail: deistler@tuwien.ac.at

${ }^{\ddagger}$ Research Group of Econometrics and System Theory, Department of Mathematical Methods in Economics, Vienna UT, E-mail: alexander.filler@tuwien.ac.at

$\S$ Research Group of Econometrics and System Theory, Department of Mathematical Methods in Economics, Vienna UT, E-mail: bernd.funovits@tuwien.ac.at
} 
To be more explicit, here we do not consider solutions of (1.1) with general white noise inputs, but construct the inputs from the outputs as their one-step-ahead forecast errors.

We call an AR system regular if $\Sigma>0$ holds, otherwise an AR system is called singular. We can write

$$
\Sigma=b b^{\prime}
$$

where $b \in \mathbb{R}^{s \times r}, \operatorname{rk}(\Sigma)=r$ and where $b$ is unique up to post-multiplication by orthogonal matrices. Accordingly, (1.1) can be written as

$$
a(z) y_{t}=b \varepsilon_{t}, b \in \mathbb{R}^{s \times r}
$$

where $\left(\varepsilon_{t}\right)$ is white noise with $\mathbb{E} \varepsilon_{t} \varepsilon_{t}^{\prime}=I$.

Singular AR systems naturally occur in linear dynamic factor models [2], [5], [8]. Linear dynamic factor models are used to compress information contained in highdimensional time series both in time and cross-sectional dimension. In such models the observations are split into one part called latent variables, representing the comovements between the observed variables, and into a noise part representing the individual movements. The latent variables result from a linear dynamic transformation of a lower dimensional dynamic factor process, which can be chosen as white noise. Thus, the latent variables have a singular spectral density showing that co-movements between the variables exist. Under suitable assumptions the latent variables may also be obtained from a linear static transformation of a static factor process. In general the dimension of the static factor process will be larger or equal to the dynamic factor process, but of smaller dimension than the latent variables. As can be shown, a static factor process has the same dynamics as the latent variables, see [5].

Moreover, it can be shown, [1], that in an appropriate rational setting the AR case is generic, i.e. the stable mini-phase factor of the spectral density of the latent variables has generically no zeros.

The paper is organized as follows: In section II we discuss stationary solutions of AR systems, which in general consist of a linearly singular and a linearly regular part. In section III we deal with the Yule-Walker equations, which may have non-unique solutions. In section IV we choose a canonical representative as a particular unique solution of the Yule-Walker equations.

2. Solutions of AR systems. A solution of (1.3) (on $\mathbb{Z}$ ) is a stochastic process $\left(y_{t} \mid t \in \mathbb{Z}\right)$ satisfying $(1.3)$ for given $(a(z), b)$ and $\left(\varepsilon_{t}\right)$. It is well known and easy to see that the set of all solutions of (1.3) consists of one particular solution plus the set of all solutions of the homogeneous equation

$$
a(z) y_{t}=0 \text {. }
$$


A special particular solution, depending only on the transfer function $a^{-1}(z) b$ and $\left(\varepsilon_{t}\right)$ will be discussed below.

Clearly, the solution set is not changed by premultiplying (1.3) by a constant non-singular matrix. In this sense, the normalization

(A4) $a_{0}=I$

is no restriction of generality and will be used throughout the paper. Also note, that, due to (A1), for any initial conditions $y_{-1}, \ldots, y_{-p}$, a forward solution on $\mathbb{N}_{0}=$ $\{0,1,2, \ldots\}$ can be calculated by iteration.

(A1) implies, but is not equivalent to $\operatorname{det} a(z) \not \equiv 0$; the latter is equivalent to say that there are no redundant equations in (1.3).

Without imposing (A3), stationary non-causal solutions may occur. However, this is ruled out by (A3). If the stability condition

$$
\operatorname{det} a(z) \neq 0,|z| \leq 1
$$

holds, then $a^{-1}(z)$ can be expanded into a power series converging in a disc containing the unit circle

$$
a^{-1}(z)=\sum_{j=0}^{\infty} h_{j} z^{j}
$$

Note that (2.3), defined in this way, gives a causal and stationary solution

$$
y_{t}^{r}=\sum_{j=0}^{\infty} k_{j} \varepsilon_{t-j}
$$

To be more general, note that the pair $(a(z), b)$ is always left co-prime for regular AR systems, but not necessarily so for singular AR systems. However, (see [3]) there always exist left co-prime $(\tilde{a}(z), b)$ such that $a^{-1}(z) b=\tilde{a}^{-1}(z) b$ holds and the degree of $\tilde{a}(z)$ is smaller than or equal to the degree of $a(z)$. The zeros of $\tilde{a}$ are the poles of the transfer function $a^{-1}(z) b=\sum_{j=0}^{\infty} k_{j} z^{j}$. From the discussion above it is clear that if

$$
\operatorname{det} \tilde{a}(z) \neq 0,|z| \leq 1
$$

holds, then a stationary solution (2.4) exists and this solution is causal and stable Note that $\operatorname{det} \tilde{a}(z)$ is independent of the special choice of the left co-prime pair $(\tilde{a}(z), b)$ and that $\operatorname{det} \tilde{a}(z)$ divides $\operatorname{det} a(z)$ (see e.g. [10]).

We call a (weakly) stationary solution of an AR system an AR process. Note that the solution (2.4) has a rational spectral density

$$
f_{y}(\lambda)=(2 \pi)^{-1}\left(\sum_{j=0}^{\infty} k_{j} e^{-i \lambda j}\right)\left(\sum_{j=0}^{\infty} k_{j} e^{-i \lambda j}\right)^{*}=(2 \pi)^{-1} a^{-1}\left(e^{-i \lambda j}\right) b b^{\prime}\left(a^{-1}\left(e^{-i \lambda j}\right)\right)^{*}
$$


where * denotes the conjugate transpose. The spectral density $f_{y}$ is regular if the AR system is regular and singular if the AR system is singular.

Note that the spectral density (2.5) of the solution (2.4) has constant rank $r$ for all $\lambda$. Thus, if we commence from the spectral density $f_{y}, f_{y}$ can be factorized (see e.g. $[12],[9])$ as

$$
f_{y}(\lambda)=k\left(e^{-i \lambda}\right) k^{*}\left(e^{-i \lambda}\right)
$$

where

$$
k(z)=\sum_{j=0}^{\infty} k_{j} z^{j}, k_{j} \in \mathbb{R}^{s \times r}
$$

is rational, has no poles for $|z| \leq 1$, and has no finite zeros at all. Thus the solution process (2.4) corresponds to Wold representation. In particular, this shows that $\left(y_{t}^{r}\right)$ is linearly regular. In addition, for given $f_{y}, k(z)$ is unique up to right multiplication by a constant orthogonal matrix.

Proposition 1. A regular AR system $(a(z), b)$ has a stationary solution (which is causal with respect to the inputs satisfying (A3)) if and only if $\operatorname{det} a(z) \neq 0,|z| \leq 1$. If a stationary solution exists, it is unique and linearly regular.

Proof. If $\operatorname{det} a(z) \neq 0,|z| \leq 1$, then a solution is given by (2.4). In this case, the homogeneous solutions consist only of exponential decays (for $t \rightarrow \infty$ ) or increasing parts (for $t \rightarrow-\infty$ ) thus a non trivial stationary solution of the homogeneous equation (2.1) does not exist.

Now assume that $\operatorname{det} a(z)$ has a zero of unit modulus. Assume that there exists a stationary solution $y_{t}$ consisting of a linearly regular $y_{t}^{r}$ and linearly singular part $y_{t}^{s}$. Applying $a(z)$ from the left on $y_{t}$, we obtain $a(z) y_{t}=a(z) y_{t}^{r}$ since the right hand side of (1.3) is linearly regular and $a(z) y_{t}^{s}$ is linearly singular. In addition the spectral density of $a(z) y_{t}^{r}$ would be singular at the frequencies corresponding to the zeros on the unit circle, whereas the spectral density of $\varepsilon_{t}$ is non-singular for all frequencies, which is a contradiction. If $\operatorname{det} a(z)$ has a zero inside of the unit circle then $a^{-1}(z)$ has no convergent power series expansion containing the unit circle and thus (A3) is not satisfied.

Proposition 2. A singular AR system $(a(z), b)$ has a stationary solution (which is causal with respect to the inputs satisfying (A3)) if and only if

$$
\operatorname{det} \tilde{a}(z) \neq 0,|z| \leq 1
$$

where $a^{-1} b=\tilde{a}^{-1} b$ and $(\tilde{a}(z), b)$ is left co-prime.

Proof. If $\operatorname{det} \tilde{a}(z) \neq 0,|z| \leq 1$ then there exists the stationary solution $y_{t}^{r}=$ 
$a^{-1}(z) b \varepsilon_{t}$. On the other hand, if $(a(z), b)$ has a stationary solution, $y_{t}$ say, then

$$
x_{t}=\left(\begin{array}{c}
y_{t} \\
\vdots \\
y_{t-p+1}
\end{array}\right)=\underbrace{\left(\begin{array}{ccccc}
a_{1} & a_{2} & \ldots & a_{p-1} & a_{p} \\
I & 0 & \ldots & \ldots & 0 \\
0 & I & 0 & & \vdots \\
\vdots & \ddots & \ddots & \ddots & 0 \\
0 & \cdots & 0 & I & 0
\end{array}\right)}_{A}\left(\begin{array}{c}
y_{t-1} \\
\vdots \\
y_{t-p}
\end{array}\right)+\underbrace{\left(\begin{array}{c}
b \\
0 \\
\vdots \\
0
\end{array}\right)}_{B}
$$

holds and thus, since $x_{t}$ is stationary too, the Ljapunov-equation

$$
\mathbb{E} x_{t} x_{t}^{\prime}=\Gamma_{x}=A \Gamma_{x} A^{\prime}+B B^{\prime}
$$

holds. Assume that $A$ is not stable, i.e. it has at least one eigenvalue $\lambda,|\lambda| \geq 1$, and a corresponding eigenvector $x$ such that

$$
\left(1-|\lambda|^{2}\right) x^{\prime} \Gamma_{x} x=x^{\prime} B B^{\prime} x .
$$

If $|\lambda|>1$ were true then for the equation (2.9) to hold $x^{\prime} \Gamma_{x} x$ has to be zero and hence $x^{\prime} B B^{\prime} x$ as well. Accordingly, if $|\lambda|=1$, it is trivial that $x^{\prime} B B^{\prime} x$ is zero. Therefore, $x^{\prime} B=0$ must hold for $|\lambda| \geq 1$. Subsequently, $\left(I-A \lambda^{-1}, B\right)$ is not of full rank or equivalently $\left(a\left(\lambda^{-1}\right), b\right)$ is not of full rank and thus not left co-prime. Let $u(z)$ be a (polynomial) greatest common left divisor of $(a(z), b)$ such that $(a(z), b)=$ $u(z)(\bar{a}(z), \bar{b}(z))$, with $(\bar{a}(z), \bar{b}(z))$ left co-prime, then $\bar{b}(z)$ is of full column rank for all $z \in \mathbb{C}$ since $a^{-1}(z) b=\bar{a}^{-1}(z) \bar{b}(z)$. Thus we find a left co-prime pair $(\tilde{a}(z), b)$ (see [5]) with $a^{-1}(z) b=\bar{a}^{-1}(z) \bar{b}(z)=\tilde{a}^{-1}(z) b$.

Now let us consider general (stationary) solutions of equation (1.3) (compare to [6]). Remember that every stationary process $y_{t}$ has, according to Wold decomposition, a uniquely defined linearly regular part $y_{t}^{r, w}$ as well as a uniquely defined linearly singular part $y_{t}^{s, w}$, where $\left(y_{t}^{r, w}\right)$ and $\left(y_{t}^{s, w}\right)$ are stationary, mutually uncorrelated and obtained by linear causal transformations of $\left(y_{t}\right)$.

THEOREM 2.1. Consider a general (stationary) solution of equation (1.3) of the form $y_{t}=a(z)^{-1} b \varepsilon_{t}+y_{t}^{h}$, where $y_{t}^{h}$ is an arbitrary solution of the homogeneous equation (2.1). Then the right hand side corresponds necessarily to Wold decomposition, i.e. $a(z)^{-1} b \varepsilon_{t}=y_{t}^{r, w}$ and $y_{t}^{h}=y_{t}^{s, w}$.

Proof. As it is immediate to see, the one-step-ahead prediction errors of $y_{t}$ and $a(z)^{-1} b \varepsilon_{t}$ are the same, as the homogeneous solution can be predicted without error. Thus $\varepsilon_{t}$ in $y_{t}^{r, w}$ and in $a(z)^{-1} b \varepsilon_{t}$ can be chosen to be the same. Let $y_{t}^{r, w}=\sum_{j=0}^{\infty} w_{j} \varepsilon_{t-j}$ and $a(z)^{-1} b \varepsilon_{t}=\sum_{j=0}^{\infty} k_{j} \varepsilon_{t-j}$, then, as $k_{0} \varepsilon_{t}$ is the one-step-ahead prediction error for $a(z)^{-1} b \varepsilon_{t}$ and $w_{0} \varepsilon_{t}$ is the one-step-ahead prediction error for $y_{t}^{r, w}$, we have $w_{0}=k_{0}$. Considering the two-step-ahead prediction errors we obtain $w_{1}=k_{1}$, and so on. Thus $y_{t}^{r, w}=a(z)^{-1} b \varepsilon_{t}$. 
REMARK 1. Note that we have not assumed a-priori that the homogeneous solution $y_{t}^{h}$ is stationary. By the proposition above however this solution has to be stationary and orthogonal to $a(z)^{-1} b \varepsilon_{t}$ whenever $y_{t}$ is stationary.

Now we consider stationary solutions of the homogeneous equation (2.1). The homogeneous equation (2.1) has (non trivial) stationary solutions if and only if det $a(z)=$ 0 for some $z$ with $|z|=1$. From the formula for the transformation of spectral measures corresponding to linear transformations of the underlying processes (see e.g. $[12])$, we obtain

$$
0=a\left(e^{-i \lambda}\right) F_{y}(d \lambda) a\left(e^{-i \lambda}\right)^{*}
$$

where $F_{y}(d \lambda)$ denotes the spectral measure of a homogeneous solution. From this it is immediate to see that a stationary solution must have a spectral distribution function which can only have jumps at frequencies $\lambda_{j}$ corresponding to the zeros of $\operatorname{det} a(z)=0$ on the unit circle and is constant elsewhere. Thus the solution must be a so called harmonic process (see [7]). A real-valued (weakly) stationary harmonic process is of the form

$$
y_{t}^{s}=\sum_{j=0}^{h} e^{-i \lambda_{j} t} w_{j}
$$

for $\mathbb{C}^{s}$ valued random values $w_{j}$ satisfying

$$
\begin{aligned}
\mathbb{E} w_{j}^{*} w_{j} & <\infty, j=1, \ldots, h \\
\mathbb{E} w_{j} & = \begin{cases}0 & \lambda_{j} \neq 0 \\
\mathbb{E} y_{t}^{s} & \lambda_{j}=0\end{cases} \\
\lambda_{1+j} & =-\lambda_{h-j} \\
w_{1+j} & =\bar{w}_{h-j}
\end{aligned}
$$

Such a process here in addition satisfies $a\left(z_{j}\right) w_{j}=0$ with $z_{j}=e^{-i \lambda_{j}}$, compare (2.10). Thus we have shown the following.

Proposition 3. For the given system (1.3) the set of all weakly stationary real valued homogeneous solutions is the set of all harmonic processes of the form (2.11) such that $a\left(z_{i}\right) w_{i}=0, \forall z_{i}$ with $\operatorname{det} a\left(z_{i}\right)=0,\left|z_{i}\right|=1$.

Now, from (2.11) we directly obtain

$$
\left(\begin{array}{c}
y_{t-1}^{s} \\
\vdots \\
y_{t-h}^{s}
\end{array}\right)=\underbrace{\left(\begin{array}{ccc}
e^{-i \lambda_{1}(t-1)} I & \ldots & e^{-i \lambda_{h}(t-1)} I \\
\vdots & & \vdots \\
e^{-i \lambda_{1}(t-h)} I & \ldots & e^{-i \lambda_{h}(t-h)} I
\end{array}\right)}_{V^{\prime}}\left(\begin{array}{c}
w_{1} \\
\vdots \\
w_{h}
\end{array}\right)
$$


where $V^{\prime}$ is nonsingular as the transposed of a generalized Vandermonde matrix. Thus

$$
\left(\begin{array}{c}
w_{1} \\
\vdots \\
w_{h}
\end{array}\right)=V^{\prime}-1\left(\begin{array}{c}
y_{t-1}^{s} \\
\vdots \\
y_{t-h}^{s}
\end{array}\right)
$$

3. The Yule-Walker Equations. Here we commence from an AR process, which in general consists of a linearly regular and an orthogonal linearly singular part corresponding to Wold decomposition

$$
y_{t}=y_{t}^{r}+y_{t}^{s}
$$

The Yule-Walker equations are of the form

where $\gamma_{j}=\mathbb{E} y_{t+j} y_{t}^{\prime}$ and $\Gamma_{m}=\left(\begin{array}{cccc}\gamma_{0} & \cdots & \cdots & \gamma_{m-1} \\ \vdots & \gamma_{0} & & \vdots \\ \vdots & & \ddots & \vdots \\ \gamma_{m-1}^{\prime} & \cdots & \cdots & \gamma_{0}\end{array}\right)=\Gamma_{m}^{r}+\Gamma_{m}^{s}$, where $\Gamma_{m}^{r}$ corresponds to the linearly regular part and $\Gamma_{m}^{s}$ to the linearly singular part respectively, and

$$
\Sigma_{\nu}=b b^{\prime}=\gamma_{0}-\left(a_{1}, \ldots, a_{p}\right)\left(\gamma_{1}, \ldots, \gamma_{p}\right)^{\prime}
$$

Here e.g. the first row of $\left(a_{1}, \ldots, a_{p}\right)$ gives the coefficients of the projection of $y_{t}^{(1)}$, i.e. the first element of $y_{t}$, on the space spanned by the entries of $y_{t-1}, \ldots, y_{t-p}$ in the Hilbert space of square integrable random variables and $\Sigma_{\nu}$ is the variance of the one-step-ahead forecasting error, i.e. of $\nu_{t}=y_{t}-\left(a_{1}, \ldots, a_{p}\right)\left(y_{t-1}^{\prime}, \ldots, y_{t-p}^{\prime}\right)^{\prime}$. Clearly $\left(a_{1}, \ldots, a_{p}\right)$ is uniquely determined from $\gamma_{0}, \ldots, \gamma_{p}$ if and only if $\Gamma_{p}$ is non-singular (or equivalently if all (one dimensional) components of $y_{t-1}, \ldots, y_{t-p}$ are linearly independent). In the case of singular systems, $\Gamma_{p}$ may be singular; then, for every row of $\left(a_{1}, \ldots, a_{p}\right)$ the solution set is one particular solution plus the kernel of $\Gamma_{p}$. On the other hand, $\nu_{t}$, whose components are the perpendiculars of orthogonal projections, and thus $\Sigma_{\nu}$ are always unique and $b$ is unique up to post-multiplication by constant orthogonal matrices. Note that $\nu_{t}$ is orthogonal to all $y_{s}, s<t$ (and not only to $\left.y_{t-1}, \ldots, y_{t-p}\right)$. A matrix $\left(a_{1}, \ldots, a_{p}, b\right)$ satisfying (3.2) and (3.3) (for given $\left.\gamma_{0}, \ldots, \gamma_{p}\right)$ is called a solution of the Yule-Walker equations. Note that the word solution used in this paper has two meanings, namely a solution of an AR system, which is a stationary process and a solution of the Yule-Walker equations which gives an AR system.

Proposition 4. Let the given sequence of covariances $\gamma_{0}, \ldots, \gamma_{p}$ correspond to a solution of a singular AR system, then any solution $\left(a_{1}, \ldots, a_{p}, b\right)$ of the Yule-Walker equations gives the same (true) sequence $\gamma_{j}, j \in \mathbb{Z}$. 
Proof. Let $j \geq p+1$ then $\gamma_{j}=\mathbb{E} y_{t} y_{t-j}^{\prime}=\mathbb{E}\left(a_{1} y_{t-1}+\ldots+a_{p} y_{t-p}+\nu_{t}\right) y_{t-j}^{\prime}=$ $E\left(a_{1} y_{t-1}+\ldots+a_{p} y_{t-p}\right) y_{t-j}^{\prime}$ which is the same for all solutions of the Yule-Walker equations. As $\gamma_{j}=\gamma_{-j}^{\prime}$ holds, the result follows.

As has been stated, if $\Gamma_{p}$ is singular, the solutions of the Yule-Walker equations are not unique. As can be seen from the following proposition, all solutions of the Yule-Walker equations result in the same transfer function.

Proposition 5. For given $\left(y_{t}\right)_{t \in \mathbb{Z}}$, for any two solutions $(a(z), b),(\tilde{a}(z), b)$ of the Yule-Walker equations, the transfer functions $a(z)^{-1} b$ and $\tilde{a}(z)^{-1} b$ coincide.

Proof. Let $(a(z), b)$ and $(\tilde{a}(z), b)$ denote two solutions of the Yule-Walker equations, then the original process $\left(y_{t}\right)_{t \in \mathbb{Z}}$ is a solution of both systems. By theorem 2.1 $a(z)^{-1} b \varepsilon_{t}=\tilde{a}(z)^{-1} b \varepsilon_{t}$ and thus $a(z)^{-1} b=\tilde{a}(z)^{-1} b$ holds.

4. A Canonical Form for Singular AR Systems. Note that in general for a given system its solution is not unique, in the sense that the linearly regular part is uniquely determined by the transfer function and the $\varepsilon_{t}$, but the linearly singular part in general depends on the choice of the initial values. On the other hand, a given process $y_{t}$ does not yield a unique AR system if $\Gamma_{p}$ is singular. For given covariances $\gamma_{0}, \ldots, \gamma_{p}$ consider the class of all AR systems $(a(z), b)$ for which initial values exist, such that the corresponding solution of the AR system yields again the same covariances. Here the problem of selecting a unique representative will be discussed.

We commence from an AR model

$$
y_{t}=a_{1} y_{t-1}+\ldots+a_{p} y_{t-p}+b \varepsilon_{t}
$$

where $b \varepsilon_{t}$ are the one-step-ahead prediction errors. The vector $x_{t-1}=\left(y_{t-1}^{\prime}, \ldots, y_{t-p}^{\prime}\right)^{\prime}$ is a state for the state space system

$$
\begin{aligned}
& x_{t}=A x_{t-1}+B \varepsilon_{t} \\
& y_{t}=C x_{t-1}+b \varepsilon_{t}
\end{aligned}
$$

where $A=\left(\begin{array}{cccc}a_{1} & a_{2} & \ldots & a_{p} \\ I & 0 & \ldots & 0 \\ 0 & I & 0 & \ldots \\ \vdots & \ddots & \ddots & \\ & & I & 0\end{array}\right), B=\left(\begin{array}{c}b \\ 0 \\ \vdots \\ \vdots \\ 0\end{array}\right)$ and $C=\left(a_{1}, \ldots, a_{p}\right)$. The state process $\left(x_{t}\right)$ is stationary, since $\left(y_{t}\right)$ is stationary by assumption. Now we select a state of smaller dimension in two steps.

\section{Step 1:}

Select the first basis of elements $y_{t-j}^{(i)}$ (where $y_{t-j}^{(i)}$ denotes the $i$-th element of the vector $y_{t-j}$ ) for the space spanned by the elements of $x_{t-1}$ in the Hilbert space of 
square integrable one dimensional random variables. This corresponds to selecting the first basis rows in terms of rows of $\Gamma_{p}$ for the row space of $\Gamma_{p}$. Such a selection is "nice" in the sense that if $y_{t-j-1}^{(i)}$ is contained in such a selection then also $y_{t-j}^{(i)}$, i.e. there are no "holes". This can be seen as follows. Suppose that $y_{t-j}^{(i)}$ can be expressed as a linear combination of preceding basis elements, then $y_{t-j-1}^{(i)}$ can be expressed by the same linear combination of the corresponding shifted elements and thus also by its preceding basis elements.

Let $S_{1}$ be a selector matrix such that $S_{1} x_{t-1}$ forms the basis described above.

Using the corresponding rows in $\Gamma_{p}$, a unique solution of the Yule-Walker equations, say $\left(a_{1}, \ldots, a_{p}\right)$ is obtained, where the columns of $a_{j}$ not corresponding to basis rows are set equal to zero. The corresponding $a(z)$ is uniquely defined in this way, because the elements of $S_{1} x_{t-1}$ are linearly independent.

\section{Step 2:}

Now note that we may still reduce the dimension of $S_{1} x_{t-1}$ because some of its elements may not be used in expressing any predictor, no matter what prediction horizon (actually, only prediction horizons from 1 to $p$ have to be checked). The state obtained by omitting these never used elements from the basis is denoted by $S x_{t-1}$. Again, there are no "holes": Consider

$$
\begin{aligned}
\hat{y}_{t \mid t-1} & =a_{1} y_{t-1}+\ldots+a_{p} y_{t-p} \\
\hat{y}_{t+1 \mid t-1} & =a_{1} \hat{y}_{t \mid t-1}+\ldots+a_{p} y_{t-p+1}
\end{aligned}
$$

where $\hat{y}_{t+j \mid t-1}$ denotes the predictor of $y_{t+j}$ from $y_{t-1}, y_{t-2}, \ldots$. Consider the case where e.g. $y_{t-1}^{(i)}$ is not an element of $S x_{t-1}$, then observe that

- the $j$-th column of $a_{1}$ has to be zero

- and $\hat{y}_{t+1 \mid t-1}=\left(a_{1}^{2}+a_{2}\right) y_{t-1}+\ldots$ and thus the $j$-th column of $a_{1}^{2}+a_{2}$ has to be zero etc.

Now we define indices $n_{1}, \ldots, n_{s}$ such that the selected state consists of the elements (reordered) $y_{t-1}^{(1)}, \ldots, y_{t-n_{1}}^{(1)}, \ldots, y_{t-1}^{(s)}, \ldots, y_{t-n_{s}}^{(s)}$. Note that the corresponding $a(z)$ has not been changed by the second step, because only certain zero coefficients have been removed; of course now $n_{i}$ is the degree of the $i$-th column of $a(z)$. For future reference, the AR system defined above will be denoted by $(\bar{a}(z), b)$, where $\bar{a}(z)$ is unique and $b$ is unique up to post-multiplication by orthogonal matrices.

Note that this procedure results in the same $\bar{a}(z)$ for any $\Gamma_{p}, p$ sufficiently large.

This selection procedure with state

$$
\bar{x}_{t}=S x_{t}
$$


leads to a "quasi companion form" $\bar{A}$ obtained from (4.2) by deleting the corresponding rows and columns which corresponds to the system

$$
\begin{aligned}
\bar{x}_{t} & =\bar{A} \bar{x}_{t-1}+\bar{B} \varepsilon_{t} \\
y_{t} & =\bar{C} \bar{x}_{t-1}+b \varepsilon_{t}
\end{aligned}
$$

Clearly, $\bar{a}(z)$ is a solution of the Yule-Walker equations. Note that by the definition of $\bar{x}_{t}$ we have

$$
\Gamma_{\bar{x}}=\mathbb{E} \bar{x}_{t} \bar{x}_{t}^{\prime}>0
$$

We have the following result for the system (4.5).

THEOREM 4.1. The system (4.5) is stable and reachable if and only if the YuleWalker equations contain a stable solution.

Proof. If the Yule-Walker equations contain a stable solution then the corresponding AR process must be linearly regular and thus $\Gamma_{p}$ must be equal to $\Gamma_{p}^{r}$. Thus also $\bar{x}_{t}$ is a linearly regular process and thus

$$
\Gamma_{\bar{x}}=(\bar{B}, \bar{A} \bar{B}, \ldots)(\bar{B}, \bar{A} \bar{B}, \ldots)^{\prime}
$$

and therefore by (4.6) the system (4.5) must be reachable.

On the other hand, if there is no stable solution of the Yule-Walker equations then the special solution $\bar{a}(z)$ is not stable and thus $\bar{A}$ has an eigenvalue of modulus larger than or equal to one. From (4.5) we get

$$
\Gamma_{\bar{x}}=\bar{A} \Gamma_{\bar{x}} \bar{A}^{\prime}+\bar{B} \bar{B}^{\prime}
$$

Let $x$ be an eigenvector of $\bar{A}$ corresponding to the eigenvalue $\lambda$, then

$$
\left(1-|\lambda|^{2}\right) x^{\prime} \Gamma_{\bar{x}} x=x^{\prime} \bar{B} \bar{B}^{\prime} x
$$

It follows from $|\lambda| \geq 1$ that $x^{\prime} \bar{B}=0$ and thus $(I-\bar{A} z, \bar{B})$ does not have full rank for all $z \in \mathbb{C}$.

COROLlary 4.2. The system $(\bar{a}(z), b)$ is stable and left co-prime if and only if the Yule-Walker equations contain a stable solution.

Proof. The proof easily follows from the fact that the eigenvalues of $\bar{A}$ correspond to the zeros of the determinant of a left co-prime matrix fraction description of the transfer function $\bar{a}^{-1}(z) b$ and that by construction the degree of $\operatorname{det} \bar{a}(z)$ is smaller or equal to $n=\sum_{i=1}^{s} n_{i}$.

REMARK 2. Note that the state space system (4.5) is not necessarily minimal. This is true for two reasons.

First, the usual notion of minimality of state space systems is related to observational equivalence via transfer functions and does not take into account the linearly singular 
component of the observed process. For example, if $y_{t}$ is purely linearly singular (and non-trivial) our corresponding state space system would necessarily have state dimension larger than zero but can never be reachable and thus not minimal.

Second, even if $y_{t}$ is purely linearly regular, the case in which the system (4.5) is reachable by theorem 4.1, then the system (4.5) is still not necessarily observable since in our construction we have only removed zero columns from the observability matrix. This can be seen from the following example.

EXAMPLE 1. Let

$$
y_{t}=\left[\begin{array}{l}
y_{t, 1} \\
y_{t, 2}
\end{array}\right]=\left[\begin{array}{ll}
1 / 4 & 1 / 6 \\
1 / 3 & 1 / 5
\end{array}\right] y_{t-1}+\left[\begin{array}{cc}
1 / 8 & 1 / 8 \\
1 / 8 & 1 / 8
\end{array}\right] y_{t-2}+\varepsilon_{t}
$$

be a regular stable $A R(2)$ process $\left(\Sigma=\mathbb{E} \varepsilon_{t} \varepsilon_{t}^{\prime}\right.$ regular) which can be represented as

$$
\begin{aligned}
& x_{t}=A x_{t-1}+B \varepsilon_{t} \\
& y_{t}=C x_{t-1}+\varepsilon_{t}
\end{aligned}
$$

$$
\begin{gathered}
A=\left[\begin{array}{cccc}
1 / 4 & 1 / 6 & 1 / 8 & 1 / 8 \\
1 / 3 & 1 / 5 & 1 / 8 & 1 / 8 \\
1 & 0 & 0 & 0 \\
0 & 1 & 0 & 0
\end{array}\right], B=\left[\begin{array}{c}
I \\
0
\end{array}\right], C=\left[\begin{array}{cccc}
1 / 4 & 1 / 6 & 1 / 8 & 1 / 8 \\
1 / 3 & 1 / 5 & 1 / 8 & 1 / 8
\end{array}\right] \\
x_{t}=\left[\begin{array}{c}
y_{t, 1} \\
y_{t, 2} \\
y_{t-1,1} \\
y_{t-1,2}
\end{array}\right] .
\end{gathered}
$$

It is easy to see that the system (4.8) (4.9) is reachable, but not observable as $[0,0,1$, $-1]^{\prime}$ is in the right kernel of $\left[C^{\prime}, A^{\prime} C^{\prime}, \ldots\right]^{\prime}$. As $y_{t}$ is a regular $A R(2)$ process, $\Gamma_{2}$ is regular and thus the steps 1 and 2 are irrelevant. A minimal system would be

$$
\begin{gathered}
\bar{A}=\left[\begin{array}{ccc}
1 / 4 & 1 / 6 & 1 / 8 \\
1 / 3 & 1 / 5 & 1 / 8 \\
1 & 1 & 0
\end{array}\right], \bar{B}=\left[\begin{array}{ll}
1 & 0 \\
0 & 1 \\
0 & 0
\end{array}\right], \bar{C}=\left[\begin{array}{ccc}
1 / 4 & 1 / 6 & 1 / 8 \\
1 / 3 & 1 / 5 & 1 / 8
\end{array}\right], \\
\bar{x}_{t}=\left[\begin{array}{c}
y_{t, 1} \\
y_{t, 2} \\
y_{t-1,1}+y_{t-1,2}
\end{array}\right] .
\end{gathered}
$$

5. Conclusions. In this contribution we have discussed certain aspects of multivariate singular AR systems. In particular we analyzed the set of solutions of such systems and the set of solutions of the corresponding Yule-Walker equations. Finally we suggested a special unique solution for the case where the Yule-Walker equations have no unique solution. 


\section{REFERENCES}

[1] B. D. O. Anderson And M. Deistler, Properties of Zero-free Transfer Function Matrices, SICE Journal of Control, Measurement and System Integration, 1:4 (2008), pp. 284-292.

[2] - Properties of Zero-free Spectral Matrices, IEEE Transactions on Automatic Control, 54:10 (2009), pp. 2365-2375.

[3] B. D. O. Anderson, M. Deistler, W. Chen, and A. Filler, AR models of singular spectral matrices, in the proceedings of the Conference on Decision and Control (CDC), Shanghai, 2009.

[4] W. Chen, B. D. O. Anderson, M. Deistler, And A. Filler, Solutions of Yule-Walker equations for Singular AR processes, forthcoming in Journal of Time Series Analysis, (2011).

[5] M. Deistler, B. D. O. Anderson, A. Filler, Ch. Zinner, and W. Chen, Generalized Linear Dynamic Factor Models - An approach via Singular Autoregressions, European Journal of Control, 16:3 (2010), pp. 211-224.

[6] M. Deistler and B. M. Poetscher, The Behaviour of the Likelihood Function for ARMA Models, Advances of Applied Probability, 16 (1984), pp. 843-865.

[7] M. Deistler and W. Scherrer, The Prague Lectures, Manuscript, TU Vienna, (1994).

[8] A. Filler, Generalized Dynamic Factor Models - Structure Theory and Estimation for Single Frequency and Mixed Frequency Data, PhD thesis, Vienna University of Technology, 2010.

[9] E. J. Hannan, Multiple Time Series, Wiley, 1970.

[10] E. J. Hannan and M. Deistler, The Statistical Theory of Linear Systems, Wiley, New York, 1988.

[11] Y. Inouye, Modeling of Multichannel Time Series and Extrapolation of Matrix-Valued Autocorrelation Sequences, IEEE Transactions on Acoustics, Speech and Signal Processing, 31:1 (1983), pp. 45-55.

[12] Yu. A. Rozanov, Stationary Random Processes, Holden-Day, San Francisco, 1967. 